\title{
Prevalencia de diabetes mellitus no insulinodependiente en la población rural de Durango, México
}

\author{
Jesús F. Guerrero Romero ${ }^{1}$, Martha Rodríguez Morán ${ }^{1}$ \\ y Francisco Sandoval Herrera²
}

RESUMEN El presente estudio tuvo el objetivo de determinar la prevalencia de diabetes mellitus no insulinodependiente (DMNID) en la población rural de Durango, México, y algunos de sus factores de riesgo. Tras un muestreo aleatorio, 30996 individuos de 30 años de edad o mayores fueron entrevistados en 627 comunidades rurales de Durango de noviembre de 1993 a diciembre de 1994 con el fin de obtener información sobre distintas variables sociodemográficas. A cada individuo se le tomó una muestra de sangre capilar para determinar su glicemia después de 10 a 12 horas de ayuno.

Se hicieron determinaciones de tendencia central y dispersión y se determinaron las distribuciones porcentuales de las variables estudiadas. Se hicieron comparaciones entre proporciones con la prueba de ji cuadrado, y entre medias con la prueba $\mathrm{t}$ de Student. La fuerza de las asociaciones se estimó con la razón de posibilidades. Para las proporciones se calculó el intervalo de confianza de $95 \%$ (IC95\%).

La muestra final se compuso de 31028 personas, 22890 (73,8 \%) de las cuales fueron mujeres y 8138 (26,2\%), hombres. Se detectaron 1004 casos de DMNID (3,2\%; IC95\%: 3,0 a 3,4\%), 767 en mujeres y 237 en hombres. Solamente 9,2\% de los individuos estudiados eran obesos. La mayor frecuencia de DMNID se documentó en individuos de 60 a 69 años de edad. Se identificaron antecedentes familiares de DMNID en 59,5\% de las personas con la enfermedad (IC $95 \%$ : 58,9 a 60,0\%) y en 26,3\% de las personas sanas (IC95\%: 25,8 a 26,8\%). Se cree que la poca prevalencia de DMNID encontrada en el presente estudio pudiera estar relacionada con la menor frecuencia de obesidad en la población encuestada.

La diabetes mellitus (DM) es una enfermedad heterogénea cuya prevalencia varía notablemente de una población a otra. Desde 1988, la OMS

\footnotetext{
1 Hospital General de Zona Número 1, Instituto Mexicano del Seguro Social (IMSS), Delegación Estatal Durango. Las solicitudes de separatas deben enviarse al primer autor a la siguiente dirección postal: Siqueiros 225 esq. c/Castañeda, Durango Dgo., CP 34000, México.

2 Coordinación Delegacional de IMSS-Solidaridad, Instituto Mexicano del Seguro Social, Delegación Estatal Durango, México.
}

ha compilado información estandarizada según la cual la prevalencia de esta enfermedad en adultos varía de 3 a $10 \%$. La revisión de estos datos sugiere la posibilidad de que exista una "epidemia" de DM en la población adulta, quizá relacionada con cambios en los estilos de vida y con el envejecimiento de la población (1).

En estudios realizados en México en 1964 en una zona urbana y en una muestra de obreros se estimó que la frecuencia de DM era, respectivamente, de 2,0 y 2,3\% (2,3). En México solo se han realizado dos estudios de prevalencia de DM en zonas rurales: uno en tres comunidades de la zona henequenera de Yucatán (4) y otro en la población rural de Durango (5). Las prevalencias estimadas fueron, respectivamente, de 1,3 y $2,9 \%$ en sujetos mayores de 20 años. Según datos recientes de la Encuesta Nacional de Enfermedades Crónicas (6) de México, la prevalencia de DM no insulinodependiente (DMNID) en la parte norte del país, donde se encuentra el estado de Durango, es de 7,8\%. 
Por otro lado, se desconoce la prevalencia de DM en la población rural de México, aunque los datos disponibles indican que en las unidades médicas rurales $1,2 \%$ de las primeras consultas por trastornos no transmisibles se atribuyen a DM; $0,8 \%$ de los egresos hospitalarios en el área rural se deben a DM, y 1,9\% de las defunciones se producen como consecuencia de esta enfermedad (7).

La estimación de la prevalencia de DMNID en una zona determinada es fundamental para montar programas de tratamiento adecuados, analizar los factores que afectan a la enfermedad y que se asocian con ella, y diseñar estudios epidemiológicos y políticas de salud $(8,9)$. De ahí que se realizara el presente estudio, cuyo objetivo es estimar la prevalencia de DMNID y algunos de sus factores de riesgo en la población rural de Durango, México.

\section{MATERIALES Y MÉTODOS}

Para hacer la investigación se utilizó un diseño transversal. La población del estudio estuvo integrada por los habitantes del área rural del estado de Durango, que se considera de marginación media con una economía de infrasubsistencia y subsistencia y que está constituida por poco más de un cuarto de millón de habitantes, o aproximadamente $26 \%$ de la población de todo el estado. Esta población rural marginada está distribuida en 627 comunidades y abarca $11 \%$ de todas las comunidades de Durango, 90\% de las cuales tienen menos de 250 habitantes (7).

Los profesionales que participaron en el estudio se seleccionaron de la lista de integrantes del Programa IMSSSolidaridad del estado de Durango. Antes de iniciarse la recolección de la información, se explicó a dichos participantes los objetivos del estudio y se les adiestró en técnicas de entrevista, administración de cuestionarios, obtención de muestras de sangre y medición de glucemia. Asimismo, el personal del Programa IMSS-Solidaridad y miembros de distintas organizaciones comunitarias de promoción de la salud explicaron a los habitantes de las comunidades estudiadas, durante sus visitas regulares a domicilio, cuáles eran los objetivos del estudio.

Las 627 comunidades que integraban la población rural marginada se agruparon en ocho zonas de acuerdo con su ubicación geográfica y accesibilidad. Para cada zona se designó un coordinador encargado de supervisar la obtención de información, recopilar los cuestionarios y obtener los resultados de la medición de la glucemia.

Casi $60 \%$ de la población de la zona rural estudiada vive en comunidades de 250 habitantes o menos con un promedio de 25 a 40 viviendas y sin ningún patrón específico de organización. Todas estas comunidades se incluyeron en el estudio. En las que tenían menos de 40 viviendas se visitaron todos los hogares a fin de entrevistar a los habitantes de 30 años de edad o mayores. Las comunidades con más de 40 viviendas se estructuraron en bloques de 10 viviendas, y de cada bloque se seleccionaron cinco viviendas mediante un muestreo aleatorio probabilístico. En ellas también se entrevistó a los moradores de 30 o más años de edad. Cada comunidad se visitó un mínimo de cuatro veces. En la muestra estudiada se incluyeron 10 comunidades de menos de 100 habitantes, cuyos moradores eran indígenas puros de los pueblos huichol, mexicanero $\mathrm{y}$ tepehuano.

Se incluyeron en la muestra voluntarios del grupo de 30 años de edad o mayor, que representaba aproximadamente $49 \%$ de la población de la zona estudiada. El diagnóstico de DMNID se estableció de acuerdo con los criterios de la OMS (10).

La encuesta se utilizó para obtener información relativa a edad, sexo, ingreso, número de integrantes de la familia, dieta habitual, antecedentes de migración por motivos laborales, antecedentes familiares y personales de DM y duración de la enfermedad. Asimismo, se midieron la estatura y el peso de los participantes, usando una escala métrica y una báscula portátil de tipo romano, y se calculó el índice de masa corporal (IMC), expresado como la razón de peso corporal en kilogra- mos a superficie corporal en metros, al cuadrado $\left(\mathrm{kg} / \mathrm{m}^{2}\right)$. La obesidad se definió como un IMC $\geq 30$. Las personas que no respondieron a las preguntas del cuestionario se excluyeron del estudio y se consideraron perdidas.

En virtud de la denominada economía campesina, que en la zona estudiada se caracteriza por unidades productivas de tipo familiar y se distingue por sus condiciones precarias y su deteriorado nivel de vida (7), el salario familiar semanal per cápita se calculó dividiendo la suma de los salarios semanales de todas las personas en el hogar que desempeñaban una actividad remunerada y el número de integrantes de la familia. La cifra obtenida se expresó en dólares estadounidenses (\$US) (calculados a razón de N\$ 3,6 pesos por dólar) sin usar ningún factor de conversión para compensar la diferencia en el costo de vida. El antecedente de migración por motivos laborales se estimó sobre la base de la migración a zonas urbanas industrializadas. Por añadidura, se cuantificó el porcentaje de su vida que cada emigrante había habitado en dichas zonas y esta variable se consideró factor de riesgo de DMNID cuando fue de $40 \%$ o más (11). Además, se averiguaron el tipo, la cantidad y la frecuencia de los alimentos ingeridos durante los 3 días que precedieron a la encuesta, y con una tabla de componentes nutricionales se analizó el contenido de carbohidratos, lípidos y proteínas de la dieta (12), expresado en términos del porcentaje de las calorías aportadas por cada componente.

Para medir la glucemia, se extrajeron muestras de sangre capilar tras 10 a 12 horas de ayuno. La glucemia se midió en tiras Dextrostix ${ }^{\circledR}$ con un glucómetro (Miles Inc., Tarry Town, New Jersey). Se consideró que una persona padecía DMNID si ya había sido diagnosticada y su glucemia era de $200 \mathrm{mg} / \mathrm{dL}$ o más. Las personas con glucemia entre 140 y 200 mg/dL fueron remitidas al centro médico más cercano a su localidad a fin de confirmar el diagnóstico mediante la medición de la glucemia plasmática en ayunas y de la prueba de la sobrecarga oral con $75 \mathrm{mg}$ de glucosa, según los 
criterios establecidos por la OMS (10). Estas personas solo se consideraron diabéticas después de recibir la confirmación diagnóstica del laboratorio.

Para analizar los datos, se calcularon medidas de tendencia central y dispersión y las distribuciones porcentuales de las variables estudiadas. Para las proporciones también se calculó el intervalo de confianza de 95\% (IC95\%). Con el fin de comparar porcentajes entre grupos, se aplicó la prueba de ji cuadrado. La comparación de medias se realizó mediante la $t$ de Student. La fuerza de las asociaciones entre variables se estimó con la razón de posibilidades (en inglés, odds ratio). Con objeto de controlar el posible efecto confusor de algunas variables, las $R P$ se estimaron ajustadas según la edad y el IMC se calculó mediante análisis estratificado, junto con sus correspondientes IC $95 \%$.

\section{RESULTADOS}

La muestra total se compuso de 31028 personas $(24,9 \%$ de la población de referencia del estudio), de las cuales $22890(73,8 \%)$ fueron mujeres y 8138 $(26,2 \%)$, hombres. Ello equivale a una razón mujer:hombre de 2,8:1,0. En total se detectaron 1004 casos de DMNID (3,2\%; IC95\%: 3,0\% a 3,4\%), de los cuales 767 fueron mujeres $3,3 \%$ del total de mujeres; IC95\%: 3,1\% a $3,6 \%)$ y 237 , hombres $(2,9 \%$ del total de hombres; IC95\%: 2,5\% - 3,3\%). De todos los diabéticos diagnosticados, 746 (2,4\%; IC95\%: 2,4\% a 2,6\%) habían recibido el diagnóstico antes del estudio y 258 (0,83\%; IC95\%: 0,7\% - 0,9\%), después. La razón de casos diagnosticados previamente a casos nuevos fue, por tanto, de 2,9:1,0 (cuadro 1). Solo se perdieron del estudio 217 personas que emigraron de su comunidad, es decir, $0,7 \%$ de todos los participantes.

Se incluyó en la muestra a 151 personas pertenecientes a comunidades indígenas puras. Aunque la estructura de edad de estas comunidades no difería de la estructura de la población mestiza, en este subgrupo no se encontraron casos previamente diagnosticados ni casos nuevos de DMNID.
CUADRO 1. Prevalencia de diabetes mellitus no insulinodependiente (DMNID) en la población rural marginada de Durango, México, desglosada por casos conocidos y de nueva detección, 1993-1994

\begin{tabular}{|c|c|c|c|c|c|c|c|c|}
\hline & \multirow{3}{*}{$\begin{array}{l}\text { Población } \\
\text { de la muestra } \\
\text { (No.) }\end{array}$} & \multicolumn{4}{|c|}{ Casos de DMNID } & & & \multirow[b]{3}{*}{ IC95\% } \\
\hline & & \multicolumn{2}{|c|}{ Conocidos } & \multicolumn{2}{|c|}{ Nuevos } & \multicolumn{2}{|c|}{ Total } & \\
\hline & & No. & $\%$ & No. & $\%$ & No. & $\%$ & \\
\hline Hombres & 8138 & 173 & $2,1^{\mathrm{a}}$ & 64 & $0,8^{a}$ & 237 & $2,9^{a}$ & $2,5-3,3$ \\
\hline Mujeres & 22890 & 573 & $2,5^{a}$ & 194 & $0,8^{a}$ & 767 & $3,4^{a}$ & $3,1-3,6$ \\
\hline Total & 31028 & 746 & $2,4^{b}$ & 258 & $0,8^{b}$ & 1004 & $3,2^{b}$ & $3,0-3,4$ \\
\hline
\end{tabular}

a Porcentaje de la muestra del mismo sexo.

${ }^{\mathrm{b}}$ Porcentaje de la muestra total.
La media de edad de la muestra estudiada fue de 48,6 $6 \pm 12,5$ años (recorrido: 30 a 82) y la de los pacientes de DMNID, 52,5 $\pm 8,9$ años. En el cuadro 2 aparecen la distribución por grupos de edad de los integrantes de la muestra con y sin DMNID, su distribución porcentual y la RP de padecer diabetes según la edad, tomando como grupo de comparación el de las personas de 30 a 34 años. En él se observa el aumento progresivo de la RP con el envejecimiento. La mayor prevalencia de DMNID se observó en el grupo de 60 a 69 años de edad.

Al examinarse la prevalencia de DMNID según el sexo y la edad, se constató una mayor frecuencia de la enfermedad en hombres menores de 45-49 años y de 60-64; en mujeres, el predominio se observó en todos los grupos mayores de esa edad (cuadro 3). Por otra parte, destaca la mayor frecuencia de personas con peso normal $(45 \%)$ y sobrepeso $(24 \%)$. El IMC fue de 30 o más solo en $9,2 \%$ de las personas estudiadas y tuvo una media de $23,9 \pm 7,4$. Ninguna persona con DMNID tuvo un IMC menor de 20. El cuadro 4 muestra la prevalencia de diabetes, ajustada según la edad, en personas con sobrepeso y obesidad.

La DMNID tuvo una duración media desde su diagnóstico de 10,7 \pm
CUADRO 2. Prevalencia de diabetes mellitus no insulinodependiente (DMNID), por grupos de edad, 1993-1994

\begin{tabular}{|c|c|c|c|c|c|c|c|}
\hline \multirow{2}{*}{$\begin{array}{c}\text { Edad } \\
\text { (años) }\end{array}$} & \multirow{2}{*}{$\begin{array}{c}\text { Población } \\
\text { de la } \\
\text { muestra } \\
\text { (No.) }\end{array}$} & \multicolumn{2}{|c|}{ Casos de DMNID } & \multirow[b]{2}{*}{ IC95\% } & \multirow[b]{2}{*}{$\mathrm{RP}^{\mathrm{b}}$} & \multirow[b]{2}{*}{ IC95\%c } & \multirow[b]{2}{*}{$P^{\mathrm{d}}$} \\
\hline & & No. & $\%^{a}$ & & & & \\
\hline $30-34$ & 5206 & 68 & 1,30 & $1,0-1,6$ & - & - & - \\
\hline $35-39$ & 4259 & 117 & 2,74 & $2,3-3,2$ & 2,13 & $1,56-2,91$ & $<0,0001$ \\
\hline $40-44$ & 4286 & 103 & 2,40 & $1,9-2,9$ & 1,86 & $1,35-2,56$ & $<0,0001$ \\
\hline $45-49$ & 3857 & 143 & 3,70 & $3,1-4,3$ & 2,90 & $2,15-3,93$ & $<0,0001$ \\
\hline $50-54$ & 3522 & 136 & 3,86 & $3,2-4,5$ & 3,03 & $2,23-4,11$ & $<0,0001$ \\
\hline $55-59$ & 2994 & 133 & 4,44 & $3,7-5,2$ & 3,51 & $2,58-4,77$ & $<0,0001$ \\
\hline $60-64$ & 2826 & 134 & 4,74 & $3,9-5,5$ & 3,76 & $2,77-5,10$ & $<0,0001$ \\
\hline $65-69$ & 1200 & 59 & 4,91 & $3,7-6,2$ & 3,90 & $2,70-5,65$ & $<0,0001$ \\
\hline $70-74$ & 1683 & 64 & 3,80 & $2,9-4,7$ & 2,98 & $2,08-4,28$ & $<0,0001$ \\
\hline $75-79$ & 606 & 22 & 3,63 & $2,3-5,4$ & 2,84 & $1,69-4,74$ & $<0,0001$ \\
\hline$\geq 80$ & 589 & 25 & 4,24 & $2,7-6,2$ & 3,34 & $2,04-5,45$ & $<0,0001$ \\
\hline Total & 31028 & 1004 & 3,23 & $3,0-3,4$ & - & - & - \\
\hline
\end{tabular}

a Porcentaje de la población de la muestra del mismo grupo de edad.

${ }^{b}$ Razón de posibilidades bruta, tomando como grupo de comparación el de 30-34 años.

c Intervalo de confianza de $95 \%$.

d Significación estadística basada en ji al cuadrado, tomando como grupo de comparación el de 30-34 años. 
CUADRO 3. Prevalencia de diabetes mellitus no insulinodependiente (DMNID), por edad y sexo, 1993-1994

\begin{tabular}{|c|c|c|c|c|c|c|c|c|c|}
\hline \multirow{3}{*}{$\begin{array}{l}\text { Edad } \\
\text { (años) }\end{array}$} & \multicolumn{3}{|c|}{ Hombres } & \multirow[b]{3}{*}{ IC95\% } & \multicolumn{3}{|c|}{ Mujeres } & \multirow[b]{3}{*}{ IC95\%b } & \multirow[b]{3}{*}{$P^{\mathrm{c}}$} \\
\hline & \multirow{2}{*}{$\begin{array}{l}\text { Total } \\
\text { (No.) }\end{array}$} & \multicolumn{2}{|c|}{ Con DMNID } & & \multirow{2}{*}{$\begin{array}{l}\text { Total } \\
\text { (No.) }\end{array}$} & \multicolumn{2}{|c|}{ Con DMNID } & & \\
\hline & & No. & $\%^{a}$ & & & No. & $\% a$ & & \\
\hline 30-34 & 943 & 18 & 1,90 & $1,1-3,0$ & 4263 & 50 & 1,17 & $0,8-1,5$ & NS \\
\hline 35-39 & 738 & 28 & 3,79 & $2,5-5,2$ & 3521 & 89 & 2,52 & $2,0-3,0$ & NS \\
\hline 40-44 & 792 & 15 & 1,89 & $1,0-3,1$ & 3494 & 88 & 2,51 & $2,0-3,0$ & NS \\
\hline $45-49$ & 891 & 41 & 4,60 & $3,3-6,0$ & 2966 & 102 & 3,43 & $2,8-4,1$ & NS \\
\hline $50-54$ & 972 & 26 & 2,67 & $1,7-3,7$ & 2550 & 110 & 4,31 & $3,5-5,1$ & 0,03 \\
\hline $55-59$ & 734 & 24 & 3,26 & $2,1-4,8$ & 2260 & 109 & 4,82 & $3,9-5,7$ & NS \\
\hline $60-64$ & 965 & 29 & 3,00 & $1,9-4,1$ & 1861 & 105 & 5,64 & $4,6-6,7$ & 0,002 \\
\hline $65-69$ & 525 & 24 & 4,57 & $2,9-6,7$ & 675 & 35 & 5,18 & $3,6-6,9$ & NS \\
\hline $70-74$ & 1038 & 16 & 1,54 & $0,9-25$ & 645 & 48 & 7,44 & $5,5-9,5$ & $<0,0001$ \\
\hline 75-79 & 267 & 8 & 2,99 & $1,3-5,8$ & 339 & 14 & 4,12 & $2,2-6,8$ & NS \\
\hline$\geq 80$ & 273 & 8 & 2,93 & $1,2-5,7$ & 316 & 17 & 5,37 & $3,1-8,4$ & NS \\
\hline Total & 8138 & 237 & 2,91 & $2,5-3,3$ & 22890 & 767 & 3,35 & $3,1-3,6$ & NS \\
\hline
\end{tabular}

a Porcentaje de la población de la muestra del mismo sexo y grupo de edad y con DMNID.

b Intervalo de confianza de $95 \%$.

c Significación estadística basada en ji al cuadrado.

9,6 años. Se observó que 2,2\% de los pacientes de DMNID no recibían tratamiento médico alguno.

La frecuencia de antecedentes familiares de la enfermedad en personas con DMNID fue de 59,5\% (IC95\%: $58,9 \%$ a $60,0 \%$ ) y en personas sanas, de 26,3\% (IC95\%: 25,8 a 26,8). El número de integrantes por familia fue similar en los subgrupos con y sin diabetes: $7,3 \pm 3,8$ y $7,1 \pm 3,7$, respectivamente. El ingreso familiar per cápita semanal fue de US\$ $8,0 \pm 2,7$ \$US en el subgrupo sin la enfermedad y de US\$ 5,6 $\pm 2,9$ en el de pacientes de DMNID (diferencia de medias $=2,4 \pm 0,22$; IC95\% $=2,39$ a 2,41).

El antecedente de migración por motivos laborales fue más frecuente entre los hombres y se identificó en 16,3\% (IC95\%: 15,9\% a 16,7\%) de todas las personas que integraron la muestra, $28,2 \%$ de las cuales habían vivido en comunidades urbanas industrializadas un mínimo de aproximadamente $40 \%$ de su vida. De estas personas, 7,5\% padecían DMNID. Las que tenían este antecedente mostraron una
RP de desarrollar DMNID, ajustada por edad e IMC y calculada tomando como grupo de comparación a las personas sin este antecedente, de 0,93 (IC95\%: 0,79 a 1,10).

La dieta de todas las personas estudiadas fue similar y estuvo constituida fundamentalmente por carbohidratos y lípidos. La ingesta de carne y de otras fuentes de proteína de origen animal era ocasional y se observó en menos de $15 \%$ de los encuestados. En cuanto a su composición, $60 \%$ de la dieta estaba formada por carbohidratos, $35 \%$, por lípidos, y $5 \%$, por proteínas.

\section{DISCUSIÓN}

CUADRO 4. Prevalencia de diabetes mellitus no insulinodependiente (DMNID), según índice de masa corporal (IMC), 1993-1994

\begin{tabular}{|c|c|c|c|c|c|c|}
\hline \multirow{2}{*}{$\begin{array}{c}\text { IMC } \\
\left(\mathrm{kg} / \mathrm{m}^{2}\right)\end{array}$} & \multicolumn{2}{|c|}{$\begin{array}{c}\text { Población } \\
\text { de la muestra }\end{array}$} & \multicolumn{2}{|c|}{ Casos de DMNID } & \multirow[b]{2}{*}{$1 \mathrm{C} 95 \%{ }^{\mathrm{b}}$} & \multirow[b]{2}{*}{$P^{c}$} \\
\hline & No. & $\%^{\mathrm{a}}$ & No. & $\%^{a}$ & & \\
\hline$<15$ & 551 & 1,8 & - & - & - & - \\
\hline $15-19,9$ & 6205 & 20,0 & - & - & - & - \\
\hline $20-24,9$ & 13963 & 45,0 & 99 & 0,7 & $0,6-0,8$ & - \\
\hline $25-29,9$ & 7447 & 24,0 & 693 & 9,3 & $8,6-9,9$ & $<0,0001$ \\
\hline$\geq 30$ & 2862 & 9,2 & 212 & 7,4 & $6,4-8,3$ & $<0,0001$ \\
\hline Total & 31028 & 100 & 1004 & 3,2 & $3,0-3,4$ & - \\
\hline
\end{tabular}

a Porcentaje de la muestra total.

b Intervalo de confianza de $95 \%$.

c Significación estadística basada en ji al cuadrado, tomando como grupo de comparación el de IMC de 20-24,9.
La participación de $24,9 \%$ de las pergraron la muestra y la forma aleatoria en que se seleccionaron sugieren que los datos obtenidos en este estudio sobre la prevalencia de DMNID en la población rural del estado de Durango son bastante confiables.

El área geográfica donde vive la población estudiada es de difícil acceso. La mayor parte de las comunidades se encuentran lejos de los centros urbanos y no tienen buenas redes de comunicación con ellos. Además, la sonas de 30 años o mayores que inte- 
zona carece de una infraestructura de laboratorio que permita realizar análisis básicos de muestras de sangre. Todos estos factores dificultan el traslado y análisis de muestras y, por consiguiente, las mediciones de glucemia en nuestro estudio se realizaron con sangre capilar. El uso de este método para medir la glucemia puede constituir una fuente de sesgo y desembocar en una subestimación de la prevalencia real de DMNID. Para reducir a un mínimo este sesgo, las mediciones de glucemia estuvieron a cargo de personal médico capacitado previamente para tal propósito y las técnicas aplicadas se desarrollaron bajo estricta vigilancia técnica a fin de obtener mediciones confiables (14). La clasificación de DMNID de la OMS excluye las glucemias comprendidas entre $120 \mathrm{y}$ $139 \mathrm{mg} / \mathrm{dL}$. Por tal motivo, las muestras de sangre de las personas cuyas glucemias iniciales se encontraron entre estos valores se enviaron a un laboratorio y el diagnóstico de diabetes se emitió solo después de disponer de la confirmación del mismo. Teniendo en cuenta estas medidas de control y el pequeño porcentaje de personas perdidas, es lícito suponer que se redujo al mínimo la proporción de resultados negativos falsos.

Para medir la glucemia en estudios epidemiológicos como el presente se recomienda usar una muestra de sangre entera capilar, método que se ha usado para llevar a cabo diversas investigaciones en otros países en desarrollo (13-15). En el presente estudio no se intentó estimar la prevalencia de personas con intolerancia a la glucosa, habida cuenta de que el método de medición de la glucemia empleado no es útil para diagnosticar este trastorno del metabolismo (14).

La prevalencia de DMNID estimada en este estudio es similar a la notificada por otros autores $(2,9 \%)$ en un estudio realizado en una zona rural de Durango (5) y menor de la estimada en la Encuesta Nacional de Enfermedades Crónicas en México (7,8\%) (6). En esta última, la población estudiada fue urbana y tenía entre 20 y 69 años de edad. Las diferencias entre nuestros resultados y los de la encuesta podrían atribuirse a diferencias en actividad física y en la exposición a factores dietéticos que favorecen la baja frecuencia de obesidad y sobrepeso $(12,14,15$, 16), así como al método de medición de la glucemia utilizado. Aunque en el presente estudio no se midió la actividad física, el IMC medio de la muestra estudiada fue menor de 25. Todo ello sugiere que el aumento de la prevalencia de DMNID en el medio rural ha sido prácticamente nulo si se compara con el observado en zonas urbanas. Asimismo, al interpretar estos resultados es menester tener en cuenta que en el área rural 1,2\% de las defunciones se atribuyen a DMNID (7), en tanto que en el área urbana las defunciones por esta causa oscilan entre $0,27 \%$ en personas de 35 a 44 años de edad y 4,93\% en las mayores de 75 años (17).

Como se indicara anteriormente, $10,7 \%$ de las personas con DMNID incluidas en la muestra tuvieron en común el haber pasado un mínimo de $40 \%$ de su vida en zonas urbanas industrializadas. La urbanización, calculada en términos de los años de residencia en ciudades industrializadas, es un factor de riesgo en el desarrollo de esta enfermedad y se utiliza como indicador del estilo de vida o del tiempo que una persona ha estado crónicamente expuesta a diversos factores de riesgo $(9,12)$. No obstante, en la muestra de este estudio no se detectó esta asociación, quizá porque no se midieron cambios en los estilos de vida ni en la alimentación o exposición a influencias ambientales nocivas. Tampoco se midió el grado de urbanización de las comunidades a las que dichas personas emigraron.

En una revisión de varios estudios, un grupo de investigadores (9) comprobó que la prevalencia de DMNID en localidades rurales de África y Asia y en una comunidad de indios mapuches de Chile fue menor de $3 \%$, cifra congruente con las encontradas en comunidades rurales poco desarrolladas. En el presente estudio se incluyó una submuestra de indígenas puros integrada por tepehuanos, huicholes y mexicaneros, y en ningún caso se detectó DMNID. Esta ausencia de la enfermedad podría atribuirse a facto- res ambientales o genéticos o a los hábitos alimentarios $(18,19)$.

La proporción de diabéticos que permanecen sin ser diagnosticados en la población reviste sumo interés para la planificación de servicios de salud $(9,21)$. En la zona urbana de México los casos de DMNID con diagnóstico nuevo notificados en la Encuesta Nacional de Enfermedades Crónicas fueron $31,3 \%$ (6) y en el presente estudio, 25,9\%. Frecuentemente, estas estimaciones están sujetas a la influencia de los métodos de medición de la glicemia (en sangre total o en suero) y del acceso de la población a los centros de diagnóstico (9).

En las zonas rurales estudiadas, la DMNID fue ligeramente más frecuente en las mujeres. La razón de mujeres a hombres en las zonas urbanas de México oscila entre 1,1:1,0 y $1,8: 1,0(6,21,22)$. Según estimaciones mundiales, no existe ninguna tendencia congruente en la frecuencia de la enfermedad según el sexo, si bien algunos datos apuntan hacia un exceso en la mujer (12). Se podría concluir, en general, que la mayor frecuencia de DMNID detectada en las mujeres se asocia con la presencia de factores de riesgo aún no bien entendidos, más que a factores de índole genética relacionados con el sexo. Por otra parte, en la muestra estudiada hubo un predominio de mujeres, favorecido por el elevado número de hombres que habían emigrado por motivos laborales. Esta redistribución de la estructura de edad de la población de referencia podría estar sesgando la prevalencia estimada de DMNID.

Agradecimiento. Los autores agradecen la participación, colaboración y respaldo prestados a este estudio por las siguientes personas: Juan Martínez Arias, Joel Mejorado Bretado, José Guadalupe Castañeda, Higinio Caballero Berraza, Rafael Moreno Gallegos, Luis Torres Guereca, Oscar Ureca Quintero, Gerardo Arellano del Toro, Alfredo Martínez Martínez, Fidel Solís, así como todo el personal médico y las autoridades delegacionales que apoyaron su realización. 


\section{REFERENCIAS}

1. King H, Rewers M. Diabetes in adults is now a third world problem. The WHO Ad Hoc Diabetes Working Group. Geneva: Bull World Health Organization 1991;69:643-648.

2. Zubirán S, Chávez A. Estudio epidemiológico de diabetes en la ciudad de México. Rev Invest Clin 1964;16:367-383.

3. Pérez Hidalgo C, Chávez A, Serrano O, Zubirán S. Encuesta sobre diabetes en "una muestra" de obreros. Rev Invest Clin 1964;16:3 85-395.

4. Chávez A, Balam G, Zubirán S. Estudio epidemiológico de la diabetes en tres comunidades de la zona henequenera del estado de Yucatán. Rev Invest Clin 1963;15:333-342.

5. Rivera Damm R, Bernal Gómez J. Frecuencia y algunas características epidemiológicas de la diabetes mellitus en una muestra de un grupo de obreros de una comunidad rural del estado de Durango. Rev Invest Clin 1973; 25:19-27.

6. Tapia Conyer R. Encuesta Nacional de Enfermedades Crónicas. México, DF: Secretaría de Salud; 1992.

7. Flores Alvarado A. Diagnóstico de salud en las zonas marginadas rurales de México, 1986-1991. México, DF: Instituto Mexicano del Seguro Social; 1992.

8. Sekikawa A, Tominaga M, Takahasi K, Eguchi $\mathrm{H}$, Igarashi M, Ohnuma $\mathrm{H}$, et al. Prevalence of diabetes and impaired glucose tolerance in Funagata area, Japan. Diabetes Care 1993;16: 570-574.

9. King $\mathrm{H}$, Rewers M. WHO Ad Hoc Working Group: global estimates for prevalence of diabetes mellitus and impaired glucose tolerance in adults. Diabetes Care 1993;16:157-177.
10. Harris M, Hadden W, Knowler W, Bennett $P$. International criteria for the diagnosis of diabetes and impaired glucose tolerance. Diabetes Care 1985;8:562-567.

11. Levit N, Natzenellenbogen J, Bradshaw D, Hoffman M, Bonnici F. The prevalence and identification of risk factors for NIDDM in urban Africans in Cape Town, South Africa. Diabetes Care 1993;16:601-607.

12. Norma Técnica 157 del Sistema Nacional de Salud. En: Instituto Mexicano del Seguro Social. Normas técnicas en materia de salubridad general. México: Subdirección General Médica, IMSS; 1990:142-149.

13. Quibrera-Infante $R$, Hernández-Rodríguez HG, Aradillas-Rodríguez C, GonzálezRodríguez S, Calles-Escandon J. Prevalencias de diabetes, intolerancia a la glucosa, hiperlipemia y factores de riesgo en función del nivel socioeconómico. Rev Invest Clin 1994; 46:25-36.

14. Si Quoc P, Marie-Aline Ch, Huy-Cuong N, Huy-Lien L, Anh-Tuan N, Thomas M, et al. Blood glucose distribution and prevalence of diabetes in Hanoi (Vietnam). Am J Epidemiol 1994;139:713-722.

15. Fish A, Pichardo E, Prazuck T. Prevalence and risk factors of diabetes mellitus in the rural region of Mali (West Africa): a practical approach. Diabetologica 1987;30:859-862.

16. Marshall J, Hamman R, Baxter J, Mayer E, Fulton D, Orleans M, et al. Ethnic differences in risk factors associated with the prevalence of non-insulin-dependent diabetes mellitus: the San Luis Valley Diabetes Study. Am J Epidemiol 1993;137: 706-718.
17. Guerrero-Romero JF, Rodríguez-Morán M Diabetes mellitus: un análisis de mortalidad por causa básica. Rev Med IMSS (México) 1996;34:43-48.

18. O’Dea K, Patel M, Kubish D, Hopper J, Traianedes K. Obesity and hyperlipidemia in a Central Australian aboriginal history of acculturation. Diabetes Care 1993;16:1004-1010.

19. Cowie C, Harris M, Silverman R, Johnson E, Rust K. Effect of multiple risk factors on differences between blacks and whites in the prevalence of non-insulin-dependent diabetes mellitus in the United States. Am I Epidemiol 1993;137:719-732.

20. Harris M. Undiagnosed NIDDM: clinical and public health issues. Diabetes Care 1993;16: 642-652.

21. Posadas-Romero C, Yamamoto-Kimura L, Lerman-Garber I, Zamora-González J, Fajardo-Gutiérrez A, Velázquez L, et al. The prevalence of NIDDM and associated coronary risk factors in Mexico City. Diabetes Care 1994;17:1441-1448.

22. González-Villalpando C, Stern MP, Villalpando E, Hazuda H, Haffner S. Prevalencia de diabetes e intolerancia a la glucosa en una población urbana de nivel económico bajo. Rev Invest Clin 1992;44:321-328.

Artículo recibido para publicación el 25 de octubre de 1995 y aceptado en versión revisada el 23 de enero de 1997.

ABSTRACT This study was carried out to determine the prevalence of non-insulin-dependent diabetes mellitus (NIDDM) in rural inhabitants of Durango, Mexico, and some of the risk factors for the disease. A random sample of 30996 individuals aged 30 years and over was selected from 627 rural communities in Durango. These persons were interviewed between November 1993 and December 1994 to obtain information on several sociodemographic variables. A capillary blood sample was collected from each individual to determine his or her glucose level after 10-12 hours of fasting.

Measures of central tendency and dispersion were calculated, and percentage distributions for the study variables were determined. Comparisons among proportions were made utilizing the chi-square test, and means were compared using Student's $t$ test. The strength of associations was estimated with odds ratios, and $95 \%$ confidence intervals (CI95\%) were calculated for proportions.

The final sample consisted of 31028 persons, of whom $22890(73.8 \%)$ were women and $8138(26.2 \%)$ were men. A total of 1004 cases of NIDDM were detected $(3.2 \%$; CI95\%: 3.0 to 3.4), 767 in women and 237 in men. Only $9.2 \%$ of the study population was obese. The highest frequency of NIDDM was documented in persons 60 to 69 years of age. Family history of NIDDM was identified in $59.5 \%$ of the persons with the disease (CI95\%: 58.9 to 60.0 ) and in $26.3 \%$ of the healthy persons (CI95\%: 25.8 to 26.8 ). The low prevalence of NIDDM found in this study may be related to the low level of obesity in the population surveyed. 